\title{
Malignant Cerebral Venous Sinus Thrombosis in Polycythemia
}

\author{
Rohan Mahale, Anish Mehta, Kiran Buddaraju, Abhinandan K. Shankar, Srinivasa Rangasetty \\ Department of Neurology, MS Ramaiah Medical College \& Hospital, Bangalore, Karnataka, India
}

\section{Dear Sir:}

Cerebral venous thrombosis (CVT) is a rare variety of cerebrovascular disease that can occur at any age and generally has a favorable outcome. However, poor outcome in CVT has been documented. ${ }^{1}$ It is caused by a wide range of etiologies, polycythemia is one among them. Polycythemia is a myeloproliferative disorder that is caused by a variety of etiologies. ${ }^{2}$ The occurrence of malignant CVT as a presenting manifestation of polycythemia has not been reported so far. Here, we report a 58-year-old man, who presented with status epilepticus and had mixed density lesion in left fronto-parietal region, which rapidly progressed within 24 hours to cause transtentorial herniation, requiring decompressive surgery.

A 58-year-old man was brought with history of 2 episodes of generalized tonic-clonic seizures (GTCS) with no regaining of consciousness in between the episodes of 1 day duration. There was preceding history of holocranial headache with vomiting 1 day prior, which lasted for 2 hours and subsided with analgesics. He did not have fever or loose stools. In the emergency room, he had 1 more episode of GTCS. He was a smoker and used to consume alcohol. On examination, he was stuporous. Pupils were equal but reacting sluggishly to light. There was bilateral papilledema. Gaze preference to left was present. Motor examination showed paucity of movement in right upper and lower limb with right plantar extensor response. Hematological investigations revealed high hemoglobin $(\mathrm{Hb})$ level $(22 \mathrm{~g} / \mathrm{dL})$ and a hematocrit of $57.5 \%$. Total leukocyte and platelets counts were normal. Renal, hepatic and thyroid function tests, serum elec- trolytes were normal. Computed tomography (CT) brain at admission showed mixed density lesion in left fronto-parietal region with hemispheric edema and anterior inter-hemispheric fissure bleed (Figure 1). Magnetic resonance venography (MRV) brain showed non-visualization of left transverse, sigmoid sinuses and internal jugular vein (Figure 2). Serum homocysteine, protein $\mathrm{C}, \mathrm{S}$, anti-thrombin III and factor V leiden levels were normal. Anti-phospholipid and anti-nuclear antibodies were negative. Serum erythropoietin level was normal and JAK2 mutation was negative. Abdominal ultrasonography was normal. Therapeutic phlebotomy was carried out. He was started on heparin anticoagulation and anti-edema measures (mannitol, oral glycerol).

His sensorium remained the same and repeat CT brain after 12 hours revealed increase in the size of the lesion with mass effect. Anti-edema measure was intensified and was put on ventilator for hyperventilation. He developed pupillary asymmetry within the next 12 hours. Repeat CT brain showed worsening of lesion with mass effect and midline shift suggestive of trans-tentorial herniation (Figure 1). Patient was taken up for emergency decompressive craniectomy. There was no improvement in sensorium following surgery. Post operative $\mathrm{Hb}$ level was $16 \mathrm{~g} / \mathrm{dL}$. Within the next 24 hours, his $\mathrm{Hb}$ level rose to $21 \mathrm{~g} / \mathrm{dL}$. Therapeutic phlebotomy was carried out. The expertise for endovascular intervention was not available. Patient succumbed to illness within 36 hours of surgery.

A wide range of etiologies have been implicated in the causation of CVT. Hypercoagulable disorders, like factor V Leiden mutation, presence of anticardiolipin antibody, antithrombin 

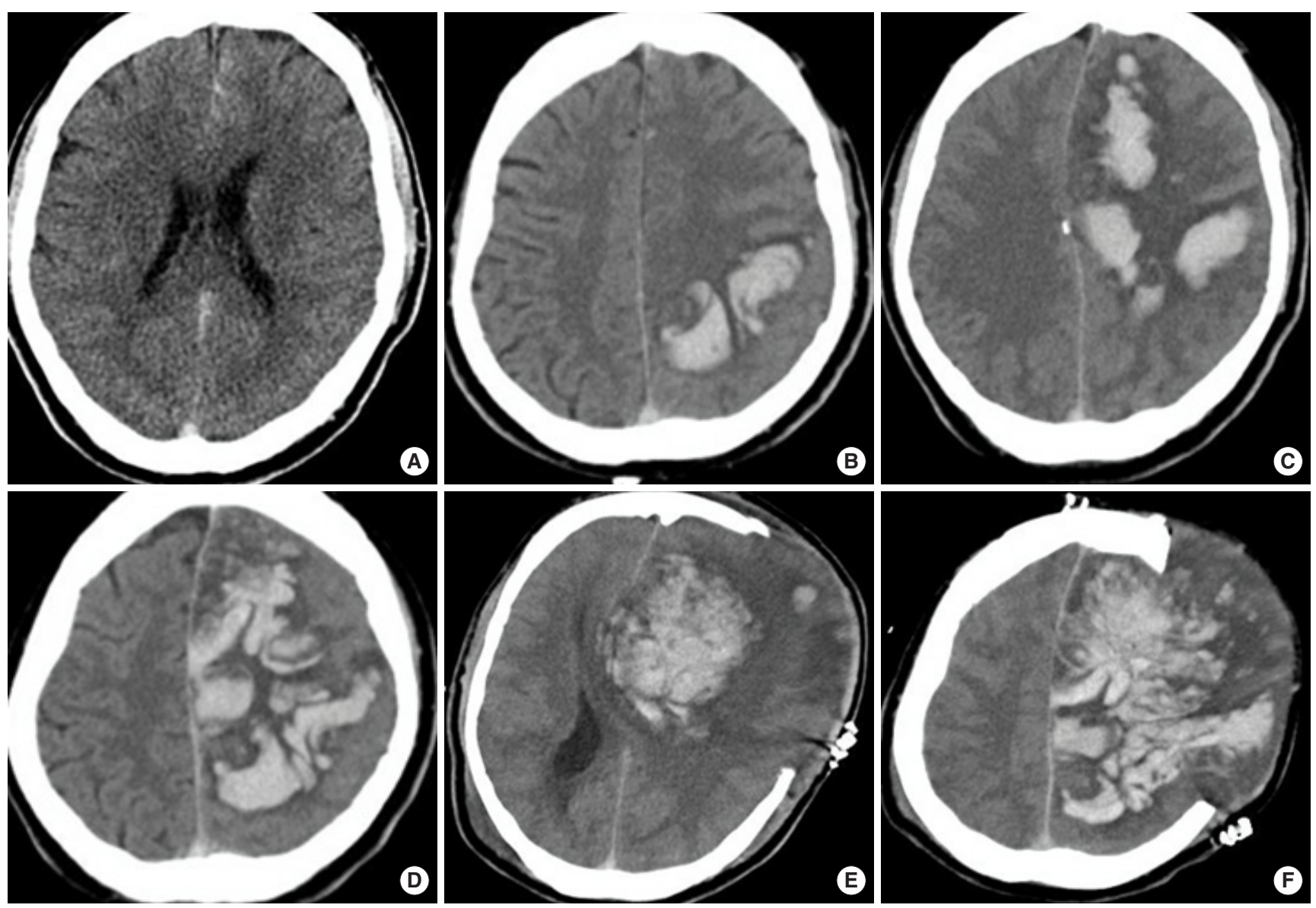

Figure 1. Non-contrast CT brain (A) \& (B) at time of admission shows bleed in anterior interhemispheric fissure (red arrow) (A); (B) hemorrhagic lesion in left frontoparietal region with hemispheric edema; (C) At 12 hours after admission shows increase in the size of lesion with mass effect; (D) At 24 hours after admission shows further worsening in the lesion; (E) \& (F) post-op CT showing mass effect and trans-tentorial herniation.

gene mutation and myeloproliferative neoplasms like polycythemia vera and essential thrombocythemia are one among them. ${ }^{2,3}$ Thrombosis is a serious complication of polycythemia and can lead to death in up to $8.3 \%$ of patients as reported by Ferro et al. ${ }^{4}$ Polycythemia can be primary (caused mainly by mutation in the JAK2 gene) or secondary. ${ }^{5}$ Chronic hypoxia causes secondary polycythemia by increasing serum erythropoietin levels leading to excess production of erythrocytes from the bone marrow. ${ }^{6}$

Polycythemia causes stasis of blood that result in hyperviscosity leading to the development of thrombosis. Thrombosis of cerebral veins or sinuses results in raised venular and capillary pressure. As local venous pressure rises, there is decrease in cerebral perfusion causing ischemic injury and cytotoxic edema. Along with cytotoxic edema, disruption of the blood-brain barrier leads to vasogenic edema, and venous and capillary rupture culminates in parenchymal hemorrhage. Thrombosis of cerebral sinuses impairs CSF absorption, leading to increased intracranial pressure. Consequently, increased intracranial pressure worsens venular and capillary hypertension and contributes to parenchymal hemorrhage and vasogenic and cytotoxic edema.?

CVT has a favorable outcome with timely diagnosis and intervention. However, unfavorable outcome do occur. Factors related to acute mortality in CVT includes advanced age, disturbed consciousness, focal neurological deficits, recurrent seizures, and hemorrhagic infarct. ${ }^{8}$ In the prospective International Study on CVT (ISCVT) cohort of 624 patients, death occurred in $8 \%$ and moderate to severe disability in $5.1 \%$ of patients, despite the use of anticoagulant treatment. ${ }^{4}$ Transtentorial herniation is the most frequent cause of death. Endovascular treatment appears to be reasonably safe and can be considered in severe cases that do not respond to heparin therapy. However, impending transtentorial herniation does not benefit from endovascular thrombolysis and needs cranial decompressive surgery. But, Coutinho et al. ${ }^{9}$ reported successful treatment of CVT with impending herniation with decompressive hemicraniectomy followed by endovascular thrombosuction.

Malignant CVT refers to supratentorial cortical lesions attributable to superficial venous system thrombosis with or without sinus involvement; with clinical or radiological signs of transten- 

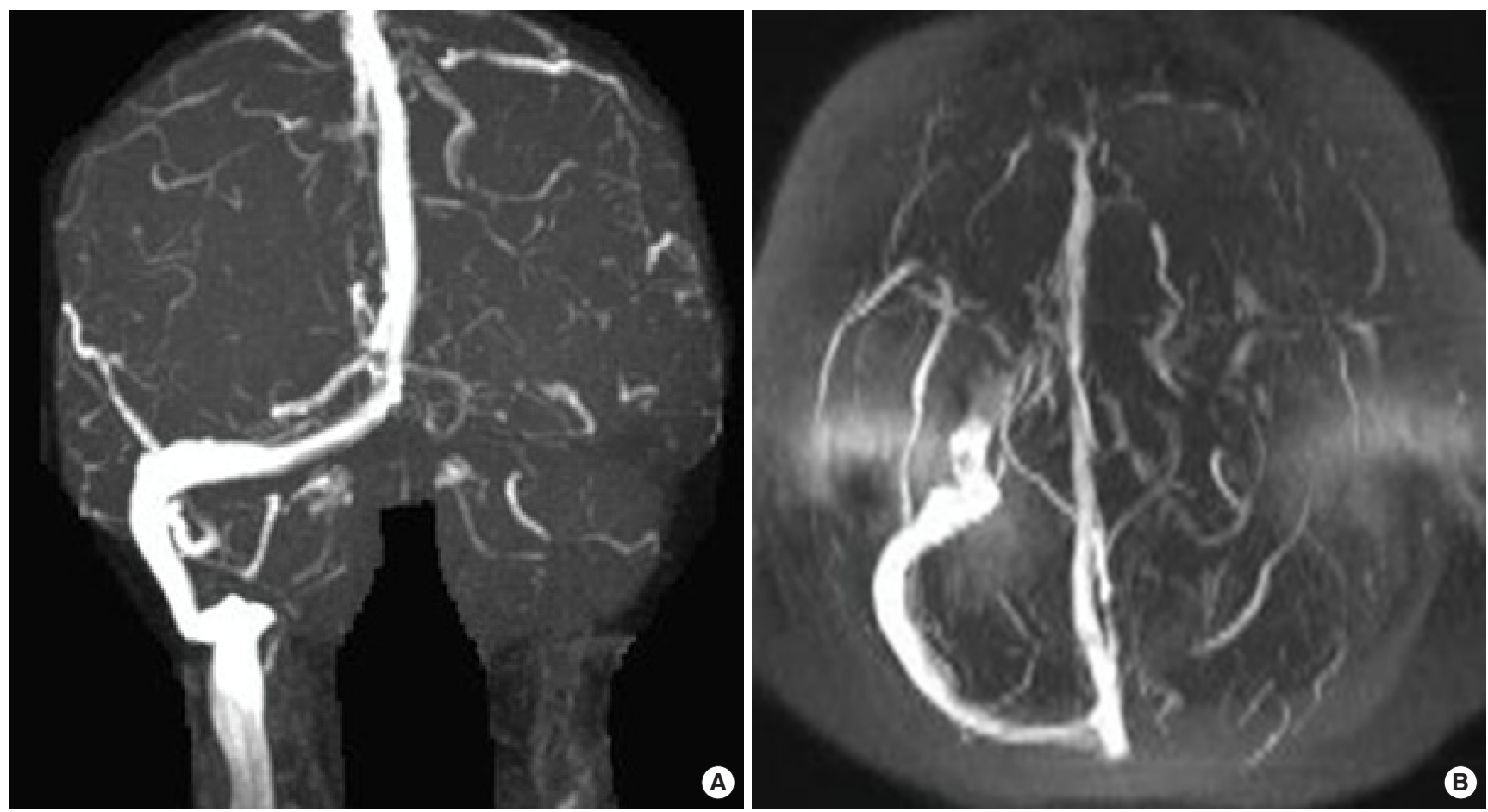

Figure 2. MRV brain (A) \& (B) showing non-visualization of left transverse, sigmoid sinuses and left internal jugular vein.

torial herniation; either at onset or after worsening despite anticoagulation. ${ }^{10}$ In a series by Théaudin et al. ${ }^{10}$ regarding the performance of decompressive surgery in malignant CVT, none of the 12 patients with malignant CVT had polycythemia as a risk factor.

The only identifiable risk factor for CVT in our patient was polycythemia. Even though JAK2 mutation results were negative, primary polycythemia cannot be ruled out as $20 \%$ of cases of polycythemia vera possess a negative JAK2 mutation, and serum erythropoietin levels were normal. Bone marrow studies could not be done in view of poor general condition. The possible etiology for polycythemia may be smoking. Smoker's polycythemia presenting as CVT is uncommon, and there is a paucity of evidence suggesting such an association. Smoker's polycythemia is diagnosed after exclusion of other causes of primary polycythemia, such as JAK2 mutation and erythropoietin level.

Polycythemia is one of the causes for occurrence of the CVT. However, the occurrence of malignant CVT in association with polycythemia has not been reported so far.

\section{References}

1. Bousser MG, Ferro JM. Cerebral venous thrombosis: an update. Lancet Neurol 2007;6:162-170.

2. Kreher S, Ochsenreither S, Trappe RU, Pabinger I, Bergmann F, Petrides PE, et al. Prophylaxis and management of venous thromboembolism in patients with myeloproliferative neoplasms: consensus statement of the Haemostasis Working Party of the German Society of Hematology and Oncology (DGHO), the Austrian Society of Hematology and Oncology (ÖGHO) and Society of Thrombosis and Haemostasis Research (GTH e.V.). Ann Hematol 2014;93:1953-1963.

3. McAloon EJ, Streiff RR, Kitchens CS. Erythrocytosis associated with carboxyhemoglobinemia in smokers. South Med J 1980;73:137-139.

4. Ferro JM, Canhão P, Stam J, Bousser MG, Barinagarrementeria F; ISCVT Investigators. Prognosis of cerebral vein and dural sinus thrombosis: results of the International Study on Cerebral Vein and Dural Sinus Thrombosis (ISCVT). Stroke 2004; 35:664-670.

5. Yoo JH, Park TS, Maeng HY, Sun YK, Kim YA, Kie JH, et al. JAK2 V617F/C618R mutation in a patient with polycythemia vera: a case study and review of the literature. Cancer Genet $C y$ togenet 2009; 189:43-47.

6. Smith JR, Landaw SA. Smokers' polycythemia. N Engl J Med 1978;298:6-10.

7. Stam J. Thrombosis of the cerebral veins and sinuses. N Engl J Med 2005;352:1791-1798.

8. Stolz E, Rahimi A, Gerriets T, Kraus J, Kaps M. Cerebral venous thrombosis: an all or nothing disease? Prognostic factors and long-term outcome. Clin Neurol Neurosurg 2005; 107:99-107.

9. Coutinho JM, Hama-Amin AD, Vleggeert-Lankamp C, Reek- 
ers JA, Stam J, Wermer MJ. Decompressive hemicraniectomy followed by endovascular thrombosuction in a patient with cerebral venous thrombosis. J Neurol 2012;259:562-564.

10. Théaudin M, Crassard I, Bresson D, Saliou G, Favrole P, Vahedi K, et al. Should decompressive surgery be performed in malignant cerebral venous thrombosis?: a series of 12 patients. Stroke 2010;41:727-731.
Correspondence: Rohan. Mahale

Department of Neurology, MS Ramaiah Medical College \& Hospital, Bangalore-560054, Karnataka, India

Tel: +91-80-40503125, Fax: +91-80-40503189

E-mail: rohanmahale83@gmail.com

Received: January 23, 2015

Revised: February 16, 2015

Accepted: April 20, 2015

Authors thank the department of neurosurgery for aiding in the surgical management of the patient.

The authors have no financial conflicts of interest. 